\title{
Immediate versus delayed insertion of the copper IUD after medical abortion at 17-20 gestational weeks: a randomized controlled trial
}

\author{
Deborah Constant ${ }^{1}$, Margit Endler ${ }^{1}$, Daniel Grossman ${ }^{2}$, Gregory Petro $^{1}$, and Malika Patel ${ }^{1}$ \\ ${ }^{1}$ University of Cape Town Faculty of Health Sciences \\ ${ }^{2}$ University of California San Francisco
}

August 23, 2020

\begin{abstract}
Objective To compare use of the copper intrauterine device (IUD) after immediate compared to delayed insertion following medical abortion (MA) at 17-20 gestational weeks (GW). Design Randomized controlled trial Setting One tertiary hospital and five community healthcare centres in South Africa Population Women admitted August 2018 to June 2019 for elective MA at 17-20 GW Methods Women were randomized to immediate (within 24 hours) or delayed (3 weeks post-abortion) insertion of the copper IUD. Follow-up was at 6 weeks, 3 and 6 months. Study outcomes were compared by intention-to-treat (ITT) and per-protocol analyses. Main outcomes Use of the original IUD, and use of any IUD at 6 weeks post-abortion (ITT) Results In the immediate $(\mathrm{n}=55)$ and delayed arms $(\mathrm{n}=57)$ respectively, $82 \%$ and $21 \%$ received the IUD as planned. By ITT, $56 \%$ in the immediate and $19 \%$ in the delayed arms were using the original IUD at six weeks $(\mathrm{p}<0.001), 76 \%$ in the immediate and $40 \%$ in the delayed arms were using any IUD $(\mathrm{p}<0.001)$. Loss-to-follow-up at 6 weeks was $2 \%$. Complete expulsion or removal occurred in $14(31 \%)$ in the immediate and $1(7 \%)$ in the delayed arms $(\mathrm{p}=0.004)$. Conclusions Insertion of an IUD immediately after MA at 17-20 GW results in increased use after 6 weeks compared to delayed insertion. Expulsion rates are higher than interval insertion and immediate insertion at earlier gestation, but similar to immediate postpartum insertion. Key words Second trimester, medical abortion, IUD Clinical trials registration: clinicaltrials.gov/ (ID NCT03505047), Pan African Trials Registry (www.pactr.org), ID PACTR201804003324963
\end{abstract}

\section{Objective}

To compare use of the copper intrauterine device (IUD) after immediate compared to delayed insertion following medical abortion (MA) at 17-20 gestational weeks (GW).

\section{Design}

Randomized controlled trial

\section{Setting}

One tertiary hospital and five community healthcare centres in South Africa

\section{Population}

Women admitted August 2018 to June 2019 for elective MA at 17-20 GW

\section{Methods}

Women were randomized to immediate (within 24 hours) or delayed (3 weeks post-abortion) insertion of the copper IUD. Follow-up was at 6 weeks, 3 and 6 months. Study outcomes were compared by intention-to-treat (ITT) and per-protocol analyses.

\section{Main outcomes}


Use of the original IUD, and use of any IUD at 6 weeks post-abortion (ITT)

\section{Results}

In the immediate $(\mathrm{n}=55)$ and delayed arms $(\mathrm{n}=57)$ respectively, $82 \%$ and $21 \%$ received the IUD as planned. By ITT, $56 \%$ in the immediate and $19 \%$ in the delayed arms were using the original IUD at six weeks $(\mathrm{p}<0.001), 76 \%$ in the immediate and $40 \%$ in the delayed arms were using any IUD ( $<<0.001)$. Loss-tofollow-up at 6 weeks was $2 \%$. Complete expulsion or removal occurred in $14(31 \%)$ in the immediate and 1 $(7 \%)$ in the delayed arms $(\mathrm{p}=0.004)$.

\section{Conclusions}

Insertion of an IUD immediately after MA at 17-20 GW results in increased use after 6 weeks compared to delayed insertion. Expulsion rates are higher than interval insertion and immediate insertion at earlier gestation, but similar to immediate postpartum insertion.

\section{Key words}

Second trimester, medical abortion, IUD

\section{Funding}

Anonymous donor (5290), National Research Foundation of South Africa /Swedish Foundation for International Cooperation in Research and Higher Education (170825260615/112631), Swedish Society of Medicine (SLS-783181), Swedish Society of Medical Research (P17-0214).

\section{Tweetable abstract}

Clinical trial shows higher use of copper IUD with immediate insertion after second trimester medical abortion

Registration:clinicaltrials.gov/ (ID NCT03505047), Pan African Trials Registry (www.pactr.org), ID PACTR201804003324963.

\section{INTRODUCTION}

Globally there is a continued high unmet need for contraception. ${ }^{1}$ Long-acting reversible contraceptives (LARC) such as the intrauterine device (IUD) are one of the most effective ways to plan and space pregnancies but women face numerous barriers to access. ${ }^{2-6}$ In South Africa, two out of three women have had an unplanned pregnancy in the previous five years with only $1.6 \%$ reportedly using an IUD. ${ }^{7}$

The availability of contraception, including LARC, is a fundamental part of post-abortion care ${ }^{8}$ Clinical trials have shown that the use of the IUD is higher after immediate compared to delayed insertion, after surgical and medical abortion in the first trimester, and after dilatation and evacuation in the second trimester, but that expulsion rates are often higher. ${ }^{9-11}$ The evidence is conflicting with respect to immediate or early post-partum insertion after vaginal delivery, with expulsion or removal rates varying between $3 \%$ and $47 \%$, but in most contexts the benefits of immediate contraception were found to outweigh the risks. ${ }^{12-14}$

It remains unclear whether the IUD can be effectively inserted after medical abortion (MA) in the second trimester. ${ }^{15}$ To our knowledge no studies have evaluated the risk-benefit of immediate insertion of the IUD after MA at 17-20 gestational weeks (GW) where higher cumulative dose of the uterotonic misoprostol is often needed.

\section{Rationale}

Women would benefit from increased access to LARC but rigorous data about optimal timing of IUD insertion after second trimester abortion are lacking.

\section{Objective}

The primary objective was to compare use of the copper IUD following immediate versus delayed insertion after a MA at 17-20 GW. The secondary objective was to compare acceptability of the IUD in these 2 groups. 


\section{METHODS}

\section{Study procedures}

We performed a randomized controlled trial (RCT) at a gynaecological department in a tertiary level teaching hospital in Cape Town, South Africa, and five affiliated community healthcare centres (CHCs) in the Cape Town metropolitan area. Eligible women were [?]18 years old, undergoing medical abortion, between 17 and $20 \mathrm{GW}$, opting and eligible for the copper IUD as post-abortion contraception. Women were informed about the study during a group contraceptive counselling session prior to their clinical evaluation at the outpatient department. They were enrolled to the study after screening for eligibility and a private written informed consent process. The research design did not involve patient-public engagement.

A research group not directly involved in study procedures generated the randomization sequence 1:1 in random permuted blocks of 4-6 and prepared sequential sealed envelopes which were delivered to the research team by hand and stored in a locked box. Two field workers performed the randomization allocation face to face with the participant after she was admitted to the gynaecological ward. The nature of the study did not permit blinding of study staff, clinical providers or participants. Between August 2018 and June 2019 we enrolled 114 women, undergoing MA between 17-20 GW with a combined mifepristone-misoprostol regimen. Prior to this, three women were enrolled to pilot study procedures.

For participants allocated to the immediate arm, clinicians planned to insert the IUD within 24 hours of completion of the abortion and prior to discharge from the gynaecological ward. The IUD was inserted by the on-call physician in an exam room in the ward or in the operating theatre if manual removal of the placenta or a vacuum aspiration for retained placenta was required. The IUD was not inserted in cases of prolonged rupture of membranes ( $>18$ hours), excessive bleeding, or signs of infection. If the patient developed a contraindication to immediate insertion, she crossed over to the delayed arm. Women in the delayed arm were referred for insertion at their referral CHC 3 weeks after the abortion. Until then they were provided 4 weeks of oral contraceptive pills. Delayed insertion was scheduled for 3 weeks post-abortion, an evidencebased protocol, and not 6 weeks as is standard practice in South Africa in order to increase compliance with the appointment. ${ }^{9}$ The IUD was inserted by a reproductive health nurse certified in abortion care and IUD insertion.

Participants were scheduled for an in-person follow-up visit including an ultrasound examination 6 weeks after the abortion with the study clinician at the tertiary hospital outpatient department. Non-returnees were contacted by phone. Two subsequent follow-up interviews took place by phone at 3 and 6 months. Participants were reminded 3 times by message and voice call to follow-up with study appointments. Those who could not be reached after this were visited at their home address. Participants received R300 compensation for in-person study visits and telephone airtime of R150 for each completed phone interview (total

\section{Outcomes}

In this study we distinguish between use of the original IUD at 6 weeks, 3 months and 6 months, and use of any IUD which includes replacement IUDs.

In the absence of core outcome sets for IUD insertion, our main outcomes were 1) use of the original IUD, and 2) use of any IUD at the end of the 6-week follow-up period.

Use of the IUD was defined as an adequately placed IUD without clinical indication for removal. We determined that the IUD was adequately placed if it had a fundal or intrauterine location on ultrasound examination and did not cause symptoms that warranted removal. If any part of the IUD was visible in the cervix it was considered intracervical and removed. IUDs with an intrauterine malposition $(>2 \mathrm{~cm}$ from the fundal endometrium) associated with symptoms of pain or abnormal discharge, were removed. If the IUD was absent upon ultrasound, and the woman had not noticed it falling out, she was referred for an abdominal $\mathrm{x}$-ray to rule out perforation and intra-abdominal location.

Our secondary outcomes were rates of complete expulsion, intracervical location, and symptomatic malpo- 
sition at 6 weeks, use of any IUD at 3 and 6 months, most effective contraception used at 3 and 6 months, cramping and bleeding at 3 and 6 months, pain on insertion, preference for immediate or delayed insertion, satisfaction with the IUD, planned duration of IUD use, and recurrent pregnancy within 6 months.

\section{Data capture and hierarchy}

We recorded outcome data from ultrasound records, follow-up interviews, and paper and electronic medical records from CHCs for the whole Cape Town Metropolitan area. If e-records showed a participant visited a healthcare facility within 6 months of the abortion, her clinical chart for this visit was reviewed for relevance with respect to study outcomes or adverse events.

The presence of an IUD at 6 weeks was primarily determined by ultrasound. If participants did not come for follow-up, self-report of use or non-use was accepted if this was not in conflict with medical records, in which case the data in medical records were assumed to be true. Participants in the delayed group with no record of IUD insertion within 3 months of the abortion at any clinic in the Cape Town Metropolitan area, and who did not report IUD insertion at a clinic outside this area, were assumed not to be using the IUD at 6 weeks or 3 months. The absence of documented IUD insertion in medical records was interpreted as non-use which minimized missing data for our main outcome in the delayed group. Secondary outcome data were captured in interviews that were sometimes cut short and not resumed, resulting in varying missing data frequencies.

\section{Analysis}

Our main outcomes were assessed after 6 weeks in an intention to treat (ITT) analysis of all eligible randomized participants $(\mathrm{n}=112)$ and a per protocol (PP) analysis of all participants that were followed up, either in person, by interview or by record review, according to the intervention received. Secondary outcomes were assessed at either 6 weeks, 3 months or 6 months post-abortion, according to ITT or PP as appropriate.

Continuous outcomes were summarized using medians and inter-quartile range for non-normal distributions and compared group-wise using Wilcoxon rank-sum (Mann-Whitney) tests. Categorical outcomes were compared using Chi-squared or Fisher's Exact test depending on the size of the subgroup. A sensitivity analysis was performed to control for uncertainty of data in cases where IUD use was assumed based on self-report. Analyses were conducted in STATA v. 15, 2019 (StataCorp. College Station, TX, USA) and significance levels are reported at $\mathrm{p}<0.05$.

\section{Sample size}

Our sample size $(\mathrm{n}=110)$ was determined in order to show a hypothesized two-fold increase ( $80 \%$ vs. $40 \%)$ in use of the IUD at 6 weeks in the immediate compared to delayed group, with a hypothesized loss-to-follow-up of $20 \%$, power set to $90 \%$, and a two-sided alpha of 0.05 and to allow for a sub-analysis of the group in each arm requiring vacuum aspiration to remove placental remains, assumed to be up to $50 \%$ of all women.

\section{RESULTS}

We screened 136 women fulfilling primary inclusion criteria. 114 eligible women were randomized, four more than planned in the protocol because four women were discontinued from the study prior to receiving their intervention. Two had a contraindication to the IUD that was not detected in the screening process, and two changed their mind regarding participation after randomization. The two women recruited by error were excluded from the analysis, the two that changed their mind were included in the ITT analysis $(\mathrm{n}=112)$. A CONSORT flowchart of participant flow through the study is shown in Figure 1.

Gestational age in the study ranged from $17 w 1 d$ to $19 w 6 d$. By ITT grouping, median gestational age was $19 \mathrm{w} 0 \mathrm{~d}$ in the immediate group and $19 \mathrm{w} 2 \mathrm{~d}$ in the delayed arm $(\mathrm{p}=0.03)$ which was assessed as clinically insignificant. All other background parameters did not differ statistically between study arms. Overall, women in the study had a median age of 28. Approximately half of participants in both study arms had not completed high school, lived in shacks and were not formally employed, one out of five reported at times 
going without food. Approximately half had given birth twice or more before. Background and reproductive characteristics of participants according to ITT and PP group are presented in Table 1.

Women received a median of 3 doses (IQR: 2-4 doses) of misoprostol before the abortion was complete (1600 ug). Median time to fetal expulsion was 6.3 hours (IQR: 4.8-9.6 hours) in the immediate arm and 8.3 hours (IQR: 6.0-11.0 hours) in the delayed arm, $40 \%$ and $60 \%$ respectively had vacuum aspiration for placental remains after the abortion. Median time from fetal expulsion to abortion completion, defined as expulsion or evacuation of the placenta, was 5.2 hours (IQR: 2.9-6.4 hours) in the immediate arm and 4.3 hours (IQR: 1.5-6.5 hours) in the delayed arm. Abortion-related variables were not significantly different between groups.

\section{Intention to treat}

The ITT analysis included all eligible randomized women $(\mathrm{n}=112), 55$ women in the immediate arm and 57 women in the delayed arm (Table 2). In the immediate arm, 45 women (82\%) received the IUD as planned. Median time from abortion completion until IUD insertion was 12.7 hours (IQR 3.8-18.5 hours). Eight women crossed over to the delayed arm because of a contraindication to immediate IUD insertion post-abortion $(n=5)$, patient request $(n=2)$ or protocol deviation $(n=1)$. Two women changed their mind about study participation and were discontinued. Out of 57 women randomized to the delayed arm, $12(21 \%)$ had the IUD inserted as planned. The remaining $45(79 \%)$ either did not go to their 3 -week appointment at the CHC $(n=35)$, went to the CHC but did not receive an IUD $(n=3)$, or changed their mind about having an IUD $(\mathrm{n}=7)$.

Use of the original IUD at 6 weeks was $56 \%$ in the immediate group and $19 \%$ in the delayed group $(\mathrm{p}<0.001)$. At the end of the 6-week follow-up period, 42 women (76\%) in the immediate arm, and $23(40 \%)$ in the delayed arm were using the original or a replacement IUD $(\mathrm{p}<0.001)$. At 3 months, use of the original, or any IUD, was $49 \%$ and $69 \%$ in the immediate group and $18 \%$ and $37 \%$ in the delayed group, respectively. Corresponding figures at 6 months were $40 \%$ and $55 \%$, and $14 \%$ and $26 \%$, respectively. Decreasing rates for use were mostly due to loss-to-follow-up (LTFU); 1 woman in the immediate arm and 1 in the delayed arm reported that they had the IUD removed between the 6 week and 6 month follow-up. Two women had a recurrent pregnancy in the 6 months following the abortion, both followed the delayed arm protocol.

In the sensitivity analysis we categorized self-reported use in the immediate group not corroborated by ultrasound ( $\mathrm{n}=2)$ as "non-use" instead of "use", and self-reported use not corroborated by medical records at 6 weeks in the delayed group as "use" instead of "non-use". This resulted in $51 \%$ and $26 \%$ ( $\mathrm{p}=0.01$ ) use of the original IUD at six weeks in the immediate arm and the delayed arm respectively. In a sub-analysis of women in the immediate arm, we found that expulsion or removal rate of the IUDs inserted after vacuum aspiration was not significantly different from those inserted without vacuum aspiration (32\% vs. $29 \%$, $\mathrm{p}=0.81$ ).

\section{Per protocol}

29 out of 43 women $(67 \%)$ who received immediate insertion and were followed up, were using the original IUD at 6 weeks, and 40 women (93\%) were using the original or a replacement IUD (Table 3). Among 65 women planned for delayed insertion per protocol, all of whom were followed up in person or by clinical record review, 14 women (22\%) had an IUD inserted and $13(20 \%)$ were still using the original IUD at 6 weeks.

Among women with immediate insertion that were not using the original IUD after six weeks, six (14\%) had a complete expulsion, and $8(18.5 \%)$ had the IUD removed because of intracervical location or symptomatic malposition. One woman with delayed insertion had the IUD removed because of symptomatic malposition.

Among women who received an IUD, $88 \%$ in the immediate group and 100\% in the delayed group were "very satisfied" with the IUD at 3 months $(\mathrm{p}=0.146)$, and $97 \%$ and $100 \%$ respectively would recommend use of the IUD to a friend $(\mathrm{p}=0.99)$ (Table S1). Most ( $85 \%$ with immediate insertion vs. $100 \%$ with delayed insertion, $\mathrm{p}=0.51$ ) planned to use the IUD for more than three years. When asked whether they would have preferred 
immediate or delayed IUD insertion if given the choice, $87 \%$ who had immediate insertion and $61 \%$ who had delayed insertion said they would have preferred immediate insertion $(\mathrm{p}=0.02)$.

\section{DISCUSSION}

\section{Main findings}

Immediate insertion of the IUD resulted in significantly higher rates of use at 6 weeks, 3 months and 6 months compared to delayed insertion, despite higher rates of expulsion or removal.

\section{Interpretation}

This study explored both efficacy measures of immediate IUD insertion at 17-20 GW, such as expulsion and removal rates, and effectiveness measures such as how immediate insertion affects continued use. Our results show that planned immediate insertion of the IUD leads to significantly more women receiving an IUD compared to planned delayed insertion. We found that four out of ten women planned for immediate IUD insertion will either not have the IUD inserted, have the IUD removed, or expel the IUD. However, if a woman receives the IUD before she leaves the hospital, it is likely that she will continue to use the original, or a replacement IUD, if provided with continuity of care, as demonstrated by a $90 \%$ continuation rate at 6 weeks among women who received immediate insertion. Both groups showed a preference for immediate insertion despite many women in the immediate group needing removal and replacement of the original IUD.

Similarly, a systematic review of immediate vs delayed IUD insertion after abortion in the first- and early second trimester concluded that immediate insertion was associated with higher expulsion rates but also with higher rates of continued use. ${ }^{11}$

Immediate insertion was associated with higher rates of expulsion and removal due to malposition than delayed insertion. Interestingly, most women with intracervical IUD placement were asymptomatic which is concerning as they may be less likely to follow-up. Two Finnish studies found expulsion/removal rates of $12.5 \%$ after early medical abortion, $27.5 \%$ after later first trimester abortion and $18.5 \%$ after early second trimester abortion although the study was underpowered for the second trimester group. ${ }^{15,16}$ Expulsion and removal rates have been estimated at $5 \%$ and $8.5 \%$ after early and late surgical abortion respectively but these studies accepted self-report of IUD use which may underestimate malposition rates. ${ }^{17,18}$ Our study did not find lower rates of expulsion and removal among women who had the IUD inserted after vacuum aspiration. It is possible that the high cumulative doses of misoprostol needed for medical abortion in the latter part of the second trimester contributed to the relatively high expulsion/malposition rates. Studies on post-placental IUD insertion also indicate that insertion within the first 10 minutes of placental delivery is more effective than insertion at 10min-48 hours, which may also be true for post-abortion insertion and which this study cannot evaluate. ${ }^{13}$

Four out of the 6 women with complete expulsion did not notice the IUD falling out and thus would be unknowingly unprotected from a repeat pregnancy had they not come for a check-up. However, women in our immediate study arm were much more likely to follow-up in-person compared to women in the delayed arm. If women who receive an IUD before leaving the hospital are strongly motivated to return, the risk associated with unnoticed expulsion would be mitigated and they would be ensured of continued contraception. Pregnancy within the first 6-week period is not impossible, and in that case an advantage of the copper- over the levonorgestrel IUD is that a new pregnancy is likely to be detected early by the absence of a period. Scheduling the first follow-up visit at 3-4 weeks may be preferable as a replacement IUD would then act as emergency contraception in case of a pregnancy occurring within that period.

The low rates of use in the delayed arm were because only one in five women presented at CHCs for IUD insertion. This was despite several pre-emptive study measures to facilitate interval insertion at CHCs compared to standard care. Interval initiation of contraception post-abortion and postpartum is known to be complicated by low compliance, which is a main rationale for quick-start contraception post-abortion and postpartum. ${ }^{9,19}$ Finally, two women who followed the delayed protocol became pregnant during the study 
period. During the post-pregnancy period women are especially vulnerable to repeat pregnancy and this must be considered in the risk-benefit calculation of immediate and delayed IUD insertion post-abortion. ${ }^{20}$

Abortion care in South Africa suffers from a scarcity of willing providers, a high rate of second trimester abortion and an overall low use of LARC. ${ }^{5}$ In contrast to this, satisfaction rates with the IUD were high throughout the study period and most women predicted that they would use the IUD for the full five years of recommended use.

\section{Strengths and limitations}

Immediate insertion of the IUD after medical abortion at 17-20 GW has not to our knowledge been studied. Most women in our study represented a group which is particularly vulnerable to infringements on their sexual and reproductive health and rights, which makes this study of particular relevance.

The study estimated sample size did not accurately predict how many women would not receive an IUD in the immediate group or how many would fail to follow-through for delayed insertion. Despite this, findings were statistically significant and loss-to-follow-up lower than predicted. Our sensitivity analysis used a conservative scenario that minimized outcome differences between groups in order to adjust for the uncertainty of missing and self-reported data and did not change the direction or significance of our findings.

The study was embedded in clinical services which currently have relatively little experience with IUD insertions which may make results less generalizable to other contexts. To mitigate this both hospital staff and CHC staff received structured mentoring sessions in IUD insertion after medical abortion prior to the study start. It is however unlikely that varying experience had a measurable impact on expulsion as expulsion rates were low with interval placement and replacement of the IUD.

Compared to a non-study situation, the study environment and other interventions may have influenced women's behaviours or opinions but they should not affect relative results. The impact of study procedures on results as well as the feasibility of the intervention is being explored in a separate process evaluation.

\section{CONCLUSIONS}

Insertion of an IUD immediately after medical abortion at 17-20 GW results in increased use after 6 weeks, 3 months and 6 months compared to delayed insertion and women show a preference for immediate insertion. Expulsion rates are higher than interval insertion and immediate insertion at earlier gestation but similar to immediate insertion after term delivery.

\section{Clinical trials registration}

Registered at clinicaltrials.gov/ (ID NCT03505047) and Pan African Trials (www.pactr.org), ID PACTR201804003324963.

\section{Disclosure of interests}

The authors report no conflict of any interests including any financial, personal, political, intellectual or religious interests.

\section{Contributions to authorship}

DC, ME, DG, MP and GP contributed to the study concept and design. DC and ME acquired funding, DC, ME and DG performed the analysis, DC and ME drafted the original manuscript. All authors reviewed and edited the final manuscript.

\section{Ethical approval}

Approved by the Human Research Ethics Committee, University of Cape Town, 5 April 2018 (HREC 007/2018) and the Western Cape Provincial Government, 18 May 2018 (WC_201804_006).

\section{Funding}


Funded by an anonymous donor foundation (5290), National Research Foundation of South Africa /Swedish Foundation for International Cooperation in Research and Higher Education (170825260615/112631), Swedish Society of Medicine (SLS-783181), and Swedish Society of Medical Research (P17-0214). Funders played no role in formulating or conducting the research, writing the paper, or the decision to publish.

\section{REFERENCES}

1. Cahill N, Sonneveldt E, Stover J, Weinberger M, Williamson J, Wei C, et al. Modern contraceptive use, unmet need, and demand satisfied among women of reproductive age who are married or in a union in the focus countries of the Family Planning 2020 initiative: a systematic analysis using the Family Planning Estimation Tool. Lancet. 2018;391(10123):870-82.

2. Committee Opinion No. 670: Immediate Postpartum Long-Acting Reversible Contraception. Obstet Gynecol. 2016;128(2) e32-e7.

3. Birgisson NE, Zhao Q, Secura GM, Madden T, Peipert JF. Preventing Unintended Pregnancy: The Contraceptive CHOICE Project in Review. Journal of Women's Health. 2105;24(5):349-53.

4. Morse J, Freedman L, Speidel JJ, Thompson KM, Stratton L, Harper CC. Postabortion contraception: qualitative interviews on counseling and provision of long-acting reversible contraceptive methods. Perspect Sex Reprod Health. 2012;44(2):100-6.

5. Masilela S. Improvement in abortion and contraception led by data-driven decisions in gauteng province, South Africa. International Journal of Gynecology and Obstetrics. 2015;2015(131):e65.

6. Harper C, Thompson K, Waxman NJ, Speidel J. Provision of long-acting reversible contraception at US abortion facilities and barriers to immediate postabortion insertion. Contraception. 2009;80(2):197-8.

7. Chersich MF, Wabiri N, Risher K, Shisana O, Celentano D, Rehle T, et al. Contraception coverage and methods used among women in South Africa: a national household survey. S Afr Med J. 2017;107(4):307-14.

8. Baldwin MK, Edelman AB. The effect of long-acting reversible contraception on rapid repeat pregnancy in adolescents: a review. J Adolesc Health. 2013;52(4 Suppl):S47-53.

9. Korjamo R, Mentula M, Heikinheimo O. Immediate versus delayed initiation of the levonorgestrel-releasing intrauterine system following medical termination of pregnancy - 1 year continuation rates: a randomised controlled trial. BJOG : an international journal of obstetrics and gynaecology. 2017;124(13):1957-64.

10. Hohmann HL, Reeves MF, Chen BA, Perriera LK, Hayes JL, Creinin MD. Immediate versus delayed insertion of the levonorgestrel-releasing intrauterine device following dilation and evacuation: a randomized controlled trial. Contraception. 2012;85(3):240-5.

11. Okusanya BO, Oduwole O, Effa EE. Immediate postabortal insertion of intrauterine devices. Cochrane Database Syst Rev. 2014(7):CD001777.

12. Cooper M, Cameron ST. Successful implementation of immediate postpartum intrauterine contraception services in Edinburgh and framework for wider dissemination. Int J Gynaecol Obstet. 2018;143:56-61.

13. Jatlaoui TC, Whiteman MK, Jeng G, Tepper NK, Berry-Bibee E, Jamieson DJ, et al. Intrauterine Device Expulsion After Postpartum Placement: A Systematic Review and Meta-analysis. Obstetrics \& Gynecology. 2018;132(4):895-905.

14. Lopez LM, Bernholc A, Hubacher D, Stuart G, Van Vliet HA. Immediate postpartum insertion of intrauterine device for contraception. Cochrane Database Syst Rev. 2015;6(CD003036).

15. Korjamo R, Mentula M, Heikinheimo O. Expulsions and adverse events following immediate and later insertion of a levonorgestrel-releasing intrauterine system after medical termination of late first- and second-trimester pregnancy: a randomised controlled trial. BJOG : an international journal of obstetrics and gynaecology. 2017;124(13):1965-72. 
16. Korjamo R, Mentula M, Heikinheimo O. Fast-track vs. delayed insertion of the levonorgestrel-releasing intrauterine system after early medical abortion - a randomized trial. Contraception. 2017;96(5):344-51.

17. Cremer M, Bullard KA, Mosley RM, Weiselberg C, Molaei M, Lerner V, et al. Immediate vs. delayed post-abortal copper T 380A IUD insertion in cases over 12 weeks of gestation. Contraception. 2011;83(6):5227 .

18. Bednarek PH, Creinin MD, Reeves MF, Cwiak C, Espey E, Jensen JT, et al. Immediate versus delayed IUD insertion after uterine aspiration. N Engl J Med. 2011;364(23):2208-17.

19. Hognert H, Kopp Kallner H, Cameron S, Nyrelli C, Jawad I, Heller R, et al. Immediate versus delayed insertion of an etonogestrel releasing implant at medical abortion - a randomized controlled equivalence trial. Human reproduction (Oxford). 2016;31(11):2484-90.

20. Appareddy S, Pryor J, Bailey B. Inter-pregnancy interval and adverse outcomes: Evidence for an additional risk in health disparate populations. J Matern Fetal Neonatal Med. 2017;30(21):2640-4.

\section{Hosted file}

Tables 1-3 for IUD study_18aug2020.docx available at https://authorea.com/users/352125/ articles/476494-immediate-versus-delayed-insertion-of-the-copper-iud-after-medicalabortion-at-17-20-gestational-weeks-a-randomized-controlled-trial

\section{Hosted file}

Fig 001 .docx available at https://authorea.com/users/352125/articles/476494-immediate-versusdelayed-insertion-of-the-copper-iud-after-medical-abortion-at-17-20-gestational-weeks-arandomized-controlled-trial 